\title{
The changes in hydrophobic-hydrophilic properties of soil organic matter under the action and aftereffect of mineral fertilizers
}

Matveeva N.V. ${ }^{1,2}$, Rogova O.B. ${ }^{1}$, Milanovsky E.Yu. ${ }^{1,2}$

${ }^{1}$ Dokuchaev Soil Science Institute, Moscow, Russia

${ }^{2}$ Lomonosov Moscow State University, Moscow Russia

doi: 10.36291/HIT.2019.matveeva.120

The contact angle of wetting (CA) of the solid phase of Chernozems under the action and aftereffect of mineral fertilizers NPK (4 years after the abolition of fertilizers) was measured. The results of this study show that CA can serve as an integral indicator of soil properties and reflect their change under anthropogenic influence. The use of mineral fertilizers NPK increases the hydrophobicity of the surface of the solid phase of the Chernozem. Three particle size and density fractions (M.S. Shaimukhametov method) were identified, different in composition: silt fraction with particles less than $1 \mu \mathrm{m}$, light fraction (LF) with a density less than $2 \mathrm{~g} / \mathrm{cm}^{3}$ and the residue after the separation of the first two fractions. Alkaline extracts of humus substances (HS) were prepared from the initial soil samples, as well as silt and LF fractions using an alkaline solution of pyrophosphate $\mathrm{Na}$. Carried out the chromatographic fractionation of alkaline extractions from soils and soil fractions of topsoil. The analysis of the obtained results shows that in the areas of application of mineral fertilizers NPK there is an increase in the content of hydrophobic components in the soil, which is consistent with the dynamics of the contact angle of wetting. Analyzing chromatograms of the soil fractions can talk about the redistribution of hydrophobic-hydrophilic components between them. More hydrophobic fragments of HS are concentrated in the LF, more hydrophilic - in the silt fraction. At the same time, the use of mineral fertilizers increases the degree of hydrophobicity of both the LF and the silt fraction. The increase in the content of hydrophobic components in the silt fraction in the application of fertilizers is more expressed than in the LF.

The site of aftereffect of mineral fertilizers is characterized by a smaller spread of the above indicators, which indicates the alignment of the hydrophobic hydrophilic properties of the soil and their fractions composition with the abolition of one of the factors of anthropogenic load. We believe that liquid chromatography of the hydrophobic interaction HS of soils and their fractions can be used in monitoring the state of the soil cover of agricultural lands.

Acknowledgements. The authors acknowledge the financial support of the Ministry of science and higher education of the Russian Federation budget project 0591-2019-0024. 